\title{
Ultralight super-hydrophobic carbon aerogels based on cellulose nanofibers/poly(vinyl alcohol)/graphene oxide (CNFs/PVA/GO) for highly effective oil-water separation
}

\author{
Zhaoyang $\mathrm{Xu}^{* 1}$, Huan Zhou ${ }^{1}$, Sicong Tan ${ }^{1}$, Xiangdong Jiang ${ }^{1}$, Weibing $\mathrm{Wu}^{2}$, \\ Jiangtao Shi ${ }^{1}$ and Peng Chen ${ }^{1}$
}

\author{
Full Research Paper

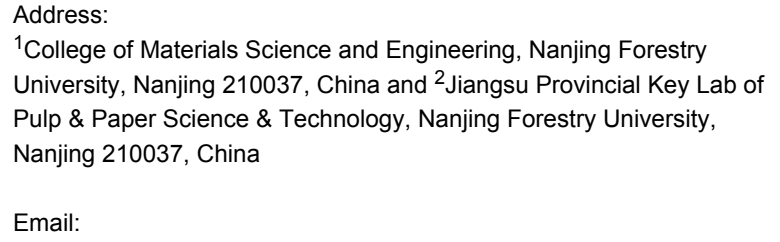

${ }^{1}$ College of Materials Science and Engineering, Nanjing Forestry University, Nanjing 210037, China and ${ }^{2}$ Jiangsu Provincial Key Lab of Pulp \& Paper Science \& Technology, Nanjing Forestry University, Nanjing 210037, China

Beilstein J. Nanotechnol. 2018, 9, 508-519. doi:10.3762/bjnano.9.49

Received: 28 September 2017

Accepted: 10 January 2018

Published: 12 February 2018

Associate Editor: B. Bhushan

(c) 2018 Xu et al.; licensee Beilstein-Institut. License and terms: see end of document.

\begin{abstract}
With the worsening of the oil-product pollution problem, oil-water separation has attracted increased attention in recent years. In this study, a porous three-dimensional (3D) carbon aerogel based on cellulose nanofibers (CNFs), poly(vinyl alcohol) (PVA) and graphene oxide (GO) was synthesized by a facile and green approach. The resulting CNF/PVA/GO aerogels were synthesized through an environmentally friendly freeze-drying process and then carbonized to yield CNF/PVA/GO carbon aerogels with low density $\left(18.41 \mathrm{mg} \mathrm{cm}^{-3}\right)$, high porosity $(98.98 \%)$, a water contact angle of $156^{\circ}$ (super-hydrophobic) and high oil absorption capacity (97 times its own weight). The carbonization treatment of the CNF/PVA/GO aerogel not only improved the hydrophobic properties but also enhanced the adsorption capacity and specific surface area. Given the many good performance characteristics and the facile preparation process of carbon aerogels, these materials are viable candidates for use in oil-water separation and environmental protection.
\end{abstract}

\section{Introduction}

In recent years, oil spills and oily industrial wastewater have attracted worldwide attention and have threatened the survival of human beings. Therefore, the development of processes and materials that can effectively separate oil and water is urgently needed. Among the existing techniques used for oil recovery, absorption is considered to be an economical choice because of its physical collection, lower costs, simplicity and high efficiency $[1,2]$. However, traditional sorbents, such as various syn- 
thetic organic polymers, natural organic materials, inorganic sorbents and polymer-based composites, tend to suffer from low adsorption capacity, secondary pollution and poor selectivity $[3,4]$. Therefore, there is a demand for the synthesis of renewable, effective and environmentally friendly sorbent materials to address the oil spill accidents.

Cellulose is considered to be the most abundant renewable natural polymer on the planet because of its biodegradability, sustainability, nontoxic nature, and biocompatibility [5]. Cellulose nanofibers (CNFs) derived from cellulose have gained wide attention due to their outstanding mechanical properties $[6,7]$ such as an elastic modulus of $140 \mathrm{GPa}$ [8]. In an aqueous environment, CNFs with a high aspect ratio and high surface area have potential for forming a 3D network structure. As a result, research on cellulose aerogels has attracted more and more attention because of its outstanding properties such as high porosity, low thermal conductivity and low density $[9,10]$. Therefore, CNFs can potentially be an excellent nanofiber for high-performance polymer nanocomposites, thereby presenting unprecedented opportunities for the development of durable engineering materials from a renewable resource for structural, consumer, and medical applications $[11,12]$. There is a long history of using plant cellulose fibers as reinforcements in polymer composite materials $[13,14]$. However, the use of nanoscale cellulose fibers to reinforce polymers is a relatively recent effort $[15,16]$. Despite the challenges described below, CNFs have been combined with various polymer matrices including thermoset resins [17,18], elastomers [19,20], polymer latexes [21], water-soluble/dispersible polymers [22,23] and biodegradable polymers [24].

Although CNF aerogels have many excellent characteristics, their strength and modulus are lower than other inorganic polymer materials. As a consequence, we report a CNF-based aerogel (CNFs/PVA/GO aerogel) composed of a CNF skeleton combined with PVA and GO, which could conquer these drawbacks. PVA is a polymer that has many excellent properties such as biodegradability, low cost, biocompatibility and water solubility [25]. Additionally, GO was chosen as the second component in the CNF-based aerogel due to its excellent mechanical properties. Besides, the oxygen atoms on its surface can promote the formation of hydrogen bonds among the three aerogel components (CNFs, PVA, and GO). The CNF/PVA/GO aerogels show outstanding thermal and mechanical properties but also exhibit some drawbacks such as poor hydrophobic properties and low oil absorption capacity. Therefore, a carbonization treatment of the CNF/PVA/GO aerogels was required to improve their properties.

In this work, we present a facile process for preparing carbon aerogels exhibiting super-hydrophobicity for oil-water separation. The CNF/PVA/GO aerogel with a macroscopic 3D structure was prepared through freeze drying to connect the three components. Then, the super-hydrophobic CNF/PVA/GO carbon aerogel was fabricated by a carbonization treatment in a tubular furnace. The as-prepared carbon aerogel exhibited a porous structure and super-hydrophobicity and showed high selectivity and outstanding oil absorption capacities for various types of organic solvents and oils. Considering its inexpensive raw materials, green synthetic method, and high performance, the CNF/PVA /GO carbon aerogel shows great potential in the treatment of oily wastewater and oil spill cleanups.

\section{Results and Discussion Morphology and microstructure of carbon aerogels}

Figure 1a and $1 \mathrm{~b}$ show digital photographs of the CNF/PVA/ GO aerogel and the CNF/PVA/GO carbon aerogel. After the carbonization treatment, the color of the CNF/PVA/GO aerogel was changed from brown to black. In addition, the dimensional contraction rate of the CNF/PVA/GO carbon aerogel was rela-
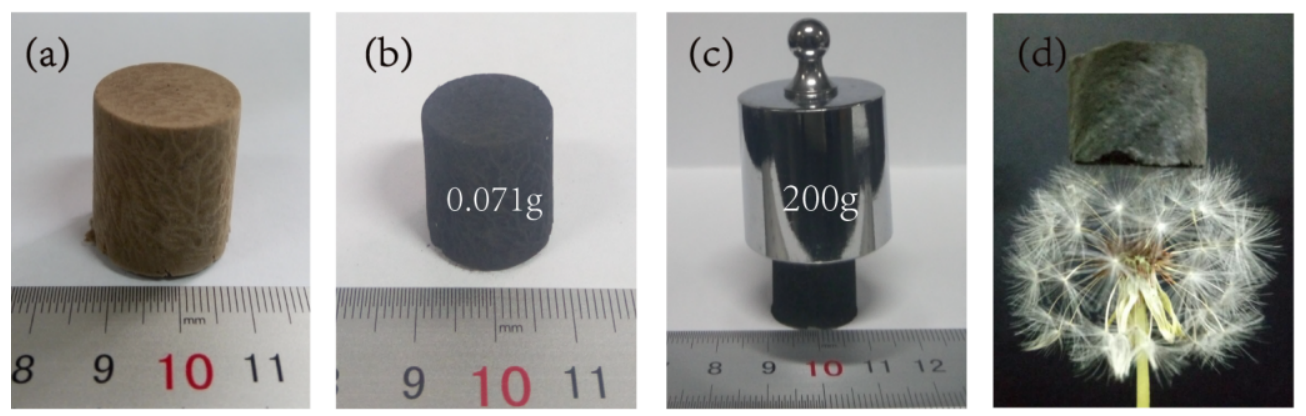

Figure 1: Digital image of (a) CNF/PVA/GO aerogel with dimensions of $1.6 \times 1.7 \mathrm{~cm}$; (b) CNF/PVA/GO carbon aerogel with dimensions of $1.5 \times 1.6 \mathrm{~cm}$; (c) the carbon aerogel supporting a mass loading of $200 \mathrm{~g}$; (d) the ultralight CNF/PVA/GO carbon aerogel, supported by a dandelion. 
tively small, which illustrated that the structure was stable. As shown in Figure 1c, the carbon aerogel, which has a weight of $0.071 \mathrm{~g}$, can support $200 \mathrm{~g}$ of weight (approximately 2817 times its weight) without any deformation, which is attributed to its interconnected 3D microstructure. The density of the carbon aerogel was approximately $18.41 \mathrm{mg} \mathrm{cm}^{-3}$, and the whole aerogel could be placed on the top of a dandelion without causing significant deformation (Figure 1d), indicating its low density.

The microstructure of the CNF/PVA/GO carbon aerogel was observed by SEM (Figure 2a, 2b, and 2d). As shown in Figure $2 \mathrm{a}$, the CNF/PVA/GO carbon aerogel showed porous and interconnected $3 \mathrm{D}$ structures with pore size ranging from $50-75 \mu \mathrm{m}$. The carbon skeleton originated from GO, and PVA and CNFs was connected to each other to form a porous hole structure. As shown in the magnified view presented in Figure 2d, the carbon skeleton was woven by numerous CNFderived crossed carbon nanofibers, so as to greatly improve its strength. The porous structure of the carbon aerogel provides sufficient space for the storage of absorbed organic solvents or oils, so that its absorption ability is greatly enhanced. In addition, the crossed CNF-derived nanofibers between the carbon skeleton can provide small pathways for liquid flow as well as enhance the capillary effect of the carbon aerogels, thus contributing to the high adsorption capacity [26]. As shown in the EDS element mapping (Figure 2c), the atomic content of $\mathrm{C}$ and $\mathrm{O}$ is $88.41 \%$ and $11.59 \%$, respectively (the appearance of $\mathrm{Si}$ is due to the glass specimen stage). The presence of the carbon element signal demonstrated that the carbonization treatment was complete, which is in good agreement with the FTIR and Raman data.
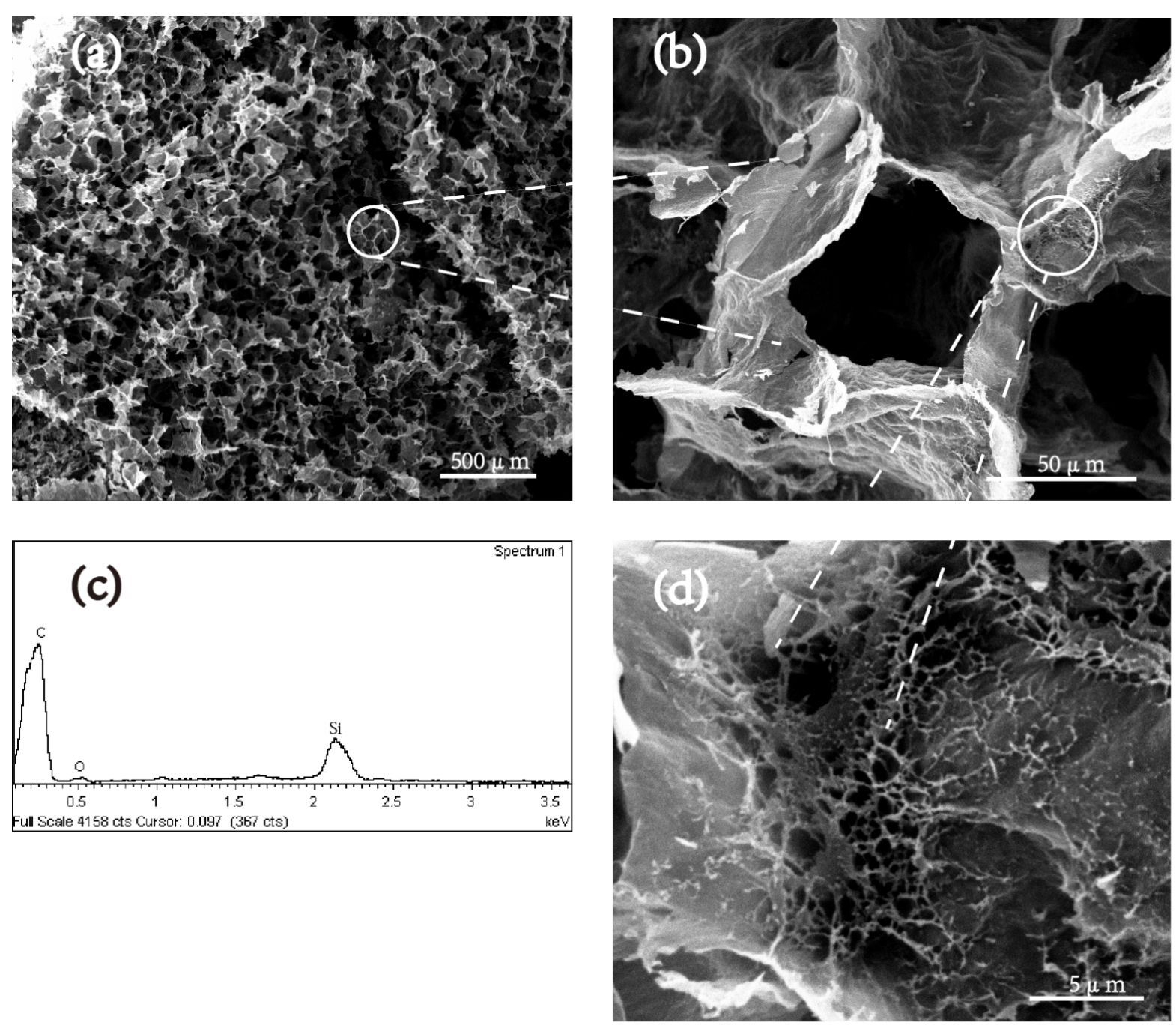

Figure 2: (a), (b), and (d) are SEM images of the inner part of the super-hydrophobic CNF/PVA/GO carbon aerogel; (c) EDS element mapping of the CNF/PVA/GO carbon aerogel. 


\section{Chemical properties}

The FTIR spectrum of the CNFs, PVA, GO, rGO, CNF/PVA/ $\mathrm{GO}$ and CNF/PVA/GO carbon aerogels are shown in Figure 3. The CNFs exhibit FTIR absorption bands around $3323 \mathrm{~cm}^{-1}$ (the stretching of hydroxy groups), $2893 \mathrm{~cm}^{-1}(\mathrm{C}-\mathrm{H}$ stretching), $1640 \mathrm{~cm}^{-1}(\mathrm{H}-\mathrm{O}-\mathrm{H}$ bending of the absorbed water), $1431 \mathrm{~cm}^{-1}$ (- $\mathrm{CH}_{2}$ bending), $1369 \mathrm{~cm}^{-1}$ (O-H bending) and $1029 \mathrm{~cm}^{-1}$ (C-O-C stretching vibrations) [27]. In Figure $3 \mathrm{~b}$, pure PVA showed peaks at $3280 \mathrm{~cm}^{-1}(\mathrm{O}-\mathrm{H}$ stretching vibration of hydroxy group), $2914 \mathrm{~cm}^{-1}$ (saturated $\mathrm{CH}_{2} / \mathrm{CH}_{3}$ groups, stretching vibration), $1714 \mathrm{~cm}^{-1}(\mathrm{C}=\mathrm{O}$ stretching), $1425 \mathrm{~cm}^{-1}$ (CH stretching) and $1083 \mathrm{~cm}^{-1}$ (C-O-C stretching vibrations) [28]. In Figure $3 \mathrm{c}$, the characteristic peaks of GO, which are located at $1714 \mathrm{~cm}^{-1}, 1586 \mathrm{~cm}^{-1}$ and $1031 \mathrm{~cm}^{-1}$, are due to $\mathrm{C}=\mathrm{O}$ in carboxylic acid and carbonyl moieties, $\mathrm{C}-\mathrm{OH}$ and $\mathrm{C}-\mathrm{O}$ vibrations, respectively. As shown in the spectrum of rGO (Figure 3d), most peaks corresponding to the carbon-oxygen functional groups disappeared, which indicates the complete reduction of GO. Figure 3e shows the FTIR spectrum of the CNF/PVA/GO aerogel. Simultaneously, the intensities of all the diffraction peaks decreased remarkably, which may indicate that a strong interaction is present among the three components. After the carbonization treatment, only carbon functional groups were retained in the CNF/PVA/GO carbon aerogel (Figure 3f). This theory has been mentioned in previous studies $[19,29]$, which is in agreement with the SEM results.

FTIR can only be used to detect signals from molecules with infrared activity. Therefore, Raman spectroscopy analysis was studied to detect the stretching vibration characteristic peak of homonuclear diatomic pairs. The Raman spectrum of the CNF aerogel is shown in Figure $4 \mathrm{a}$. The bands at $2892 \mathrm{~cm}^{-1}$, $1365 \mathrm{~cm}^{-1}, 1097 \mathrm{~cm}^{-1}$ and $889 \mathrm{~cm}^{-1}$, were due to the asymmetric and symmetric $\mathrm{CH}_{2}$ stretching vibrations, the $\mathrm{CH}_{2}$ deformation vibrations, the $\mathrm{C}-\mathrm{O}-\mathrm{C}$ glycosidic link asymmetric stretching modes and the in-plane symmetric stretching of C-O-C. In previous work, a similar Raman spectrum was observed for cotton cellulose [30]. Figure $4 \mathrm{~b}$ shows the Raman spectrum of PVA films. The strong band at $2911 \mathrm{~cm}^{-1}$ is due to symmetric and asymmetric $\mathrm{CH}_{2}$ stretching vibrations. The Raman spectra of GO and rGO are presented in Figure $4 \mathrm{c}$ and Figure $4 \mathrm{~d}$, respectively, showing the tangential G-band at $1587 \mathrm{~cm}^{-1}$ and the disorder-induced D-band at $1347 \mathrm{~cm}^{-1}$ [31] As is well-known, the G-band corresponds to the tangential vibration of the carbon atoms, while the D-band corresponds to unordered carbon or defective graphitic structures in a Raman spectrum for carbon materials. Besides, the relative intensities of the D-band and G-band $\left(I_{\mathrm{D}} / I_{\mathrm{G}}\right)$ of GO and $\mathrm{rGO}$ are 0.91 and 1.09 , respectively. After the GO was reduced to $\mathrm{rGO}$, a large number of $\mathrm{sp}^{3}$ hybrid carbon atoms deoxidized to form a new

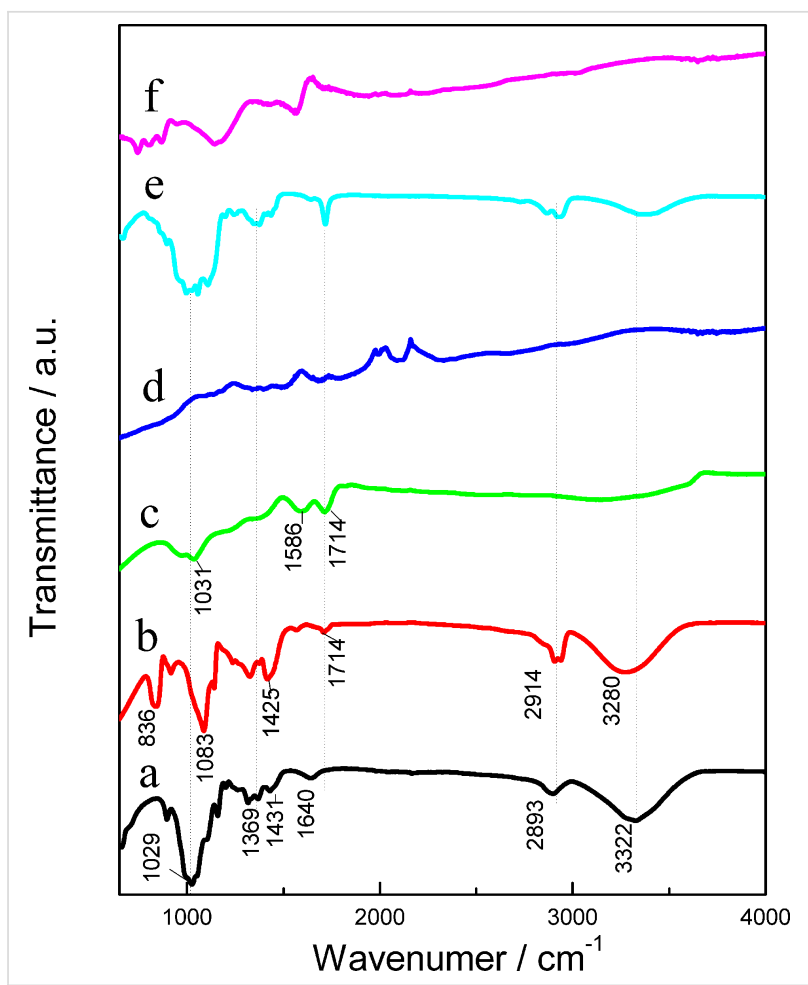

Figure 3: FTIR spectra of the (a) CNFs, (b) PVA, (c) GO, (d) rGO, (e) CNF/PVA/GO aerogel, and (f) CNF/PVA/GO carbon aerogel.

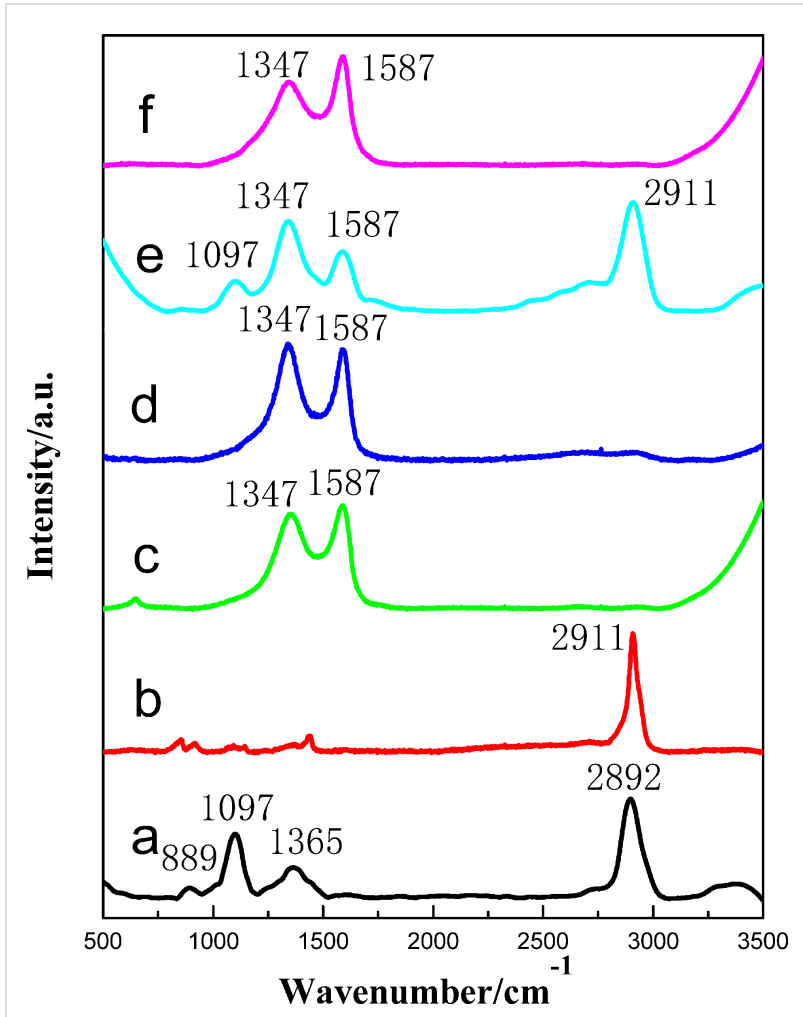

Figure 4: Raman images of the (a) CNFs, (b) PVA, (c) GO, (d) rGO, and (e) CNF/PVA/GO aerogels and the (f) CNF/PVA/GO carbon aerogel. 
$\mathrm{sp}^{2}$ hybridization area. However, the $\mathrm{sp}^{2}$ area of rGO is smaller than that of GO, so the average $\mathrm{sp}^{2}$ area of $\mathrm{rGO}$ decreases and the ratios increase, as reflected in the gradual $I_{\mathrm{D}} / I_{\mathrm{G}}$ enhancement observed in the Raman spectra; this result has also been reported in the literature $[32,33]$. For the $\mathrm{CNF} / \mathrm{PVA} / \mathrm{GO}$ aerogel (Figure 4e), the bands of CNFs, PVA and GO are observed in the Raman spectrum. After the carbonization treatment (Figure 4f), all characteristic bands of the carbon aerogel are tangential G-bands and the disorder-induced D-band, which are in agreement with the SEM and FTIR results.

To further confirm the reduction degree of the GO during the carbonization treatment, X-ray photoelectron spectroscopy was carried out (Figure $5 \mathrm{a}$ ). The higher $\mathrm{C} / \mathrm{O}$ ratio of rGO revealed the effective reduction during the thiolation reaction, which was verified more clearly in the high-resolution $\mathrm{C} 1 \mathrm{~s}$ core-level spectra shown in Figure $5 \mathrm{~b}$ and $5 \mathrm{c}$. The typical $\mathrm{C}$ 1s core-level spectrum was divided into several peaks including $\mathrm{C}=\mathrm{C}$ $(\approx 284.5 \mathrm{eV}), \mathrm{C}-\mathrm{C}(\approx 286.8 \mathrm{eV}), \mathrm{C}=\mathrm{O}(\approx 287.8 \mathrm{eV})$, and $\mathrm{O}-\mathrm{C}=\mathrm{O}$ $(\approx 289.1 \mathrm{eV})[34,35]$. Compared with $\mathrm{GO}$, the peak intensities of $\mathrm{C}=\mathrm{O}, \mathrm{C}-\mathrm{O}$ and $\mathrm{O}-\mathrm{C}=\mathrm{O}$ peaks of $\mathrm{rGO}$ showed an obvious decrease caused by the ring-opening of epoxide groups and nucleophilic substitution on the hydroxy groups. After the carbonization treatment, the oxygen-containing functional groups in GO were deoxygenated along with the restoration of conjugated structures. Besides, the hydroxy groups on the GO were expected to be significantly eliminated during the thermal decomposition, as was confirmed by the FTIR spectra (Figure 3). It has been reported that the binding energy of hydroxy groups to the carbon atoms is lower than the other oxygen-functional groups, so the hydroxy groups are significantly removed at the initial stage of thermal decomposition $[36,37]$. The above results and analysis indicated that rGO was successfully prepared, accompanied by a drastic decrease in the number of polar groups and a certain degree of reduction [38].

As shown in Figure 6, the thermal stability of the CNF/PVA/ GO aerogels before and after the carbon treatment, pure CNFs, pure PVA and GO were measured by thermogravimetric analysis (TGA) in nitrogen from 30 to $600{ }^{\circ} \mathrm{C}$. In addition to the $\mathrm{CNF} / \mathrm{PVA} / \mathrm{GO}$ carbon aerogels, the other three samples showed three different stages of weight loss: the water decomposition stage, polymer decomposition stage, and residual substance decomposition stage. The thermal stability of the aerogel was slightly improved after the introduction of GO. The maximum decomposition temperature of the CNFs after GO introduction
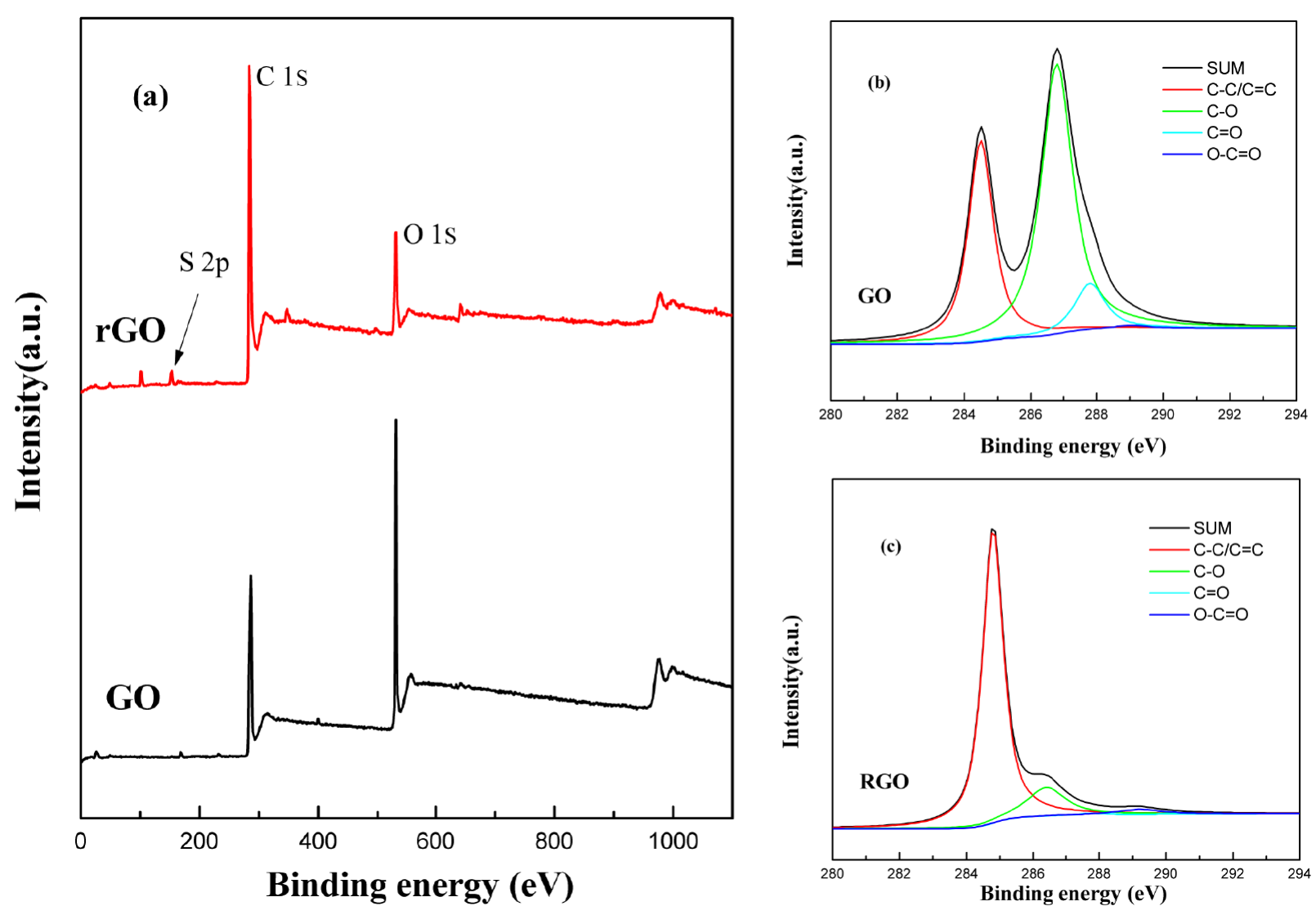

Figure 5: X-ray photoelectron spectrometry spectra and deconvolution of (a) survey scans, and C 1s high-resolution spectra of (b) GO and (c) rGO. 


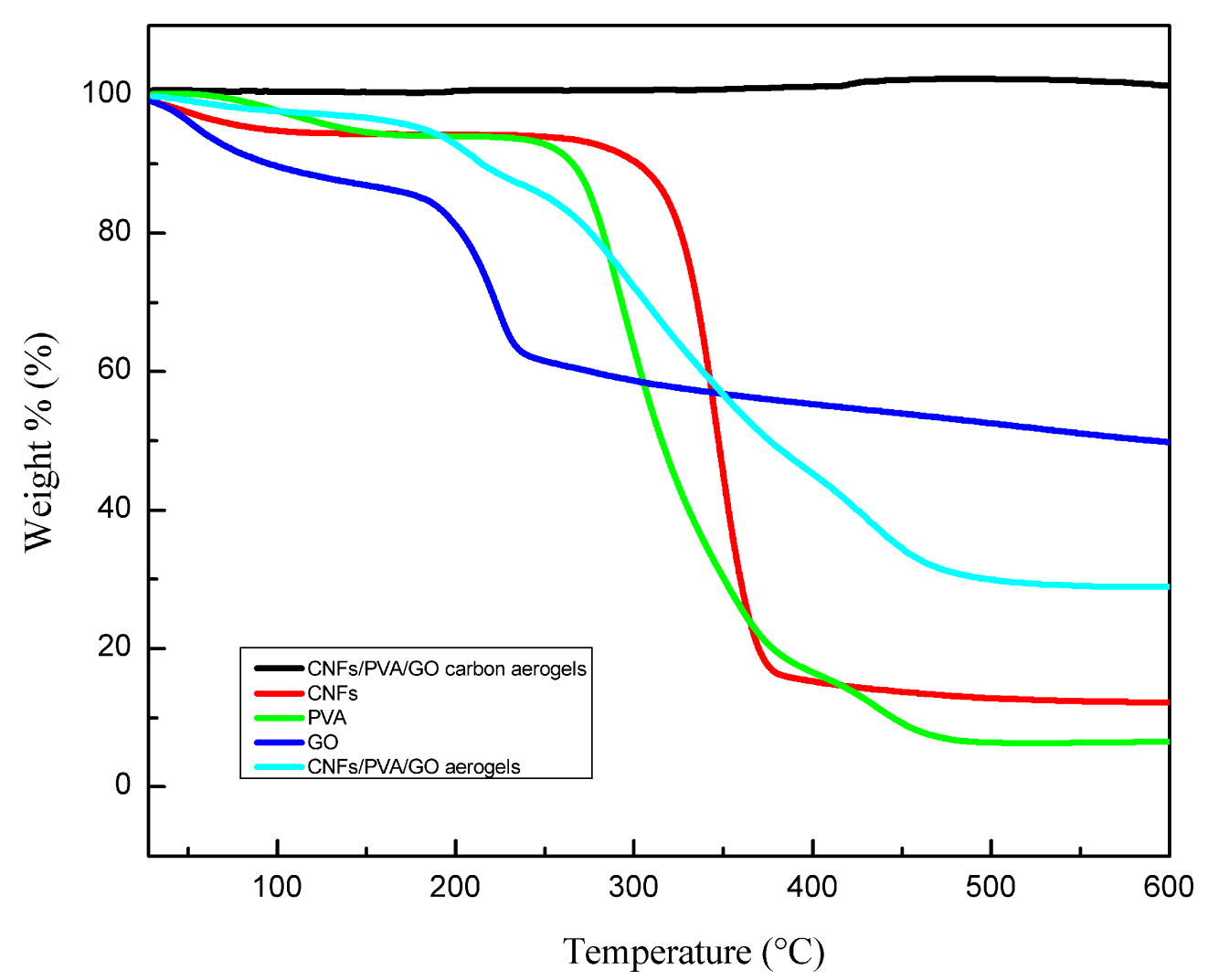

Figure 6: Thermogravimetric analysis (TGA) curves of the CNFs, pure PVA, GO, CNF/PVA/GO aerogel and the CNF/PVA/GO carbon aerogel.

increased from 237 to $465^{\circ} \mathrm{C}$, showing that the addition of GO improves the thermal stability of the composite film to some extent. This may have occurred because the GO retarded the thermal decomposition of the aerogels. As shown in the TGA curves of the CNF/PVA/GO carbon aerogels, no weight loss occurred, indicating that carbonation was complete.

Hydrophobicity is an important parameter that influences the absorption capability for organic solvents and oils. Besides, it is reported that the water contact angles are affected significantly by the hydrophilic functional groups on the aerogel surfaces. After the carbonization treatment, the hydrophilic functional groups on the carbon aerogel surfaces disappeared. As shown in Figure 7, the water contact angle of the carbon aerogel was found to be approximately $156^{\circ}$. It is found that the water contact angle on the external surfaces do not show any obvious change with time. These results further confirm the excellent super-hydrophobicity stability of the carbon aerogels examined in this work. Besides, the porous skeleton of the CNF/PVA/GO carbon aerogels provide a large volume for the storage of absorbed organic solvents/oils. As shown in Figure 8, the carbon aerogels can absorb oils (dyed with Sudan red) rapidly and then float on the surface of the water due to its low density, superoleophilic and super-hydrophobic behavior.
In addition to high oil absorption capacity, CNF/PVA/GO carbon aerogels must be easily recyclable and regenerable to be put into practical applications. Figure 9 shows the recycling process of CNF/PVA/GO carbon aerogels for absorption of soybean oil by absorption/combustion processes. During the burning process of the carbon aerogels, the basic skeleton is retained, and most of the carbon aerogel did not burn at all, demonstrating their good flame retardance. As shown in Figure 10, the absorption capacity decreases from 75 to $38.2 \mathrm{~g} / \mathrm{g}$ after 10 cycles, indicating the relatively stable recycling performance of the CNF/PVA/GO carbon aerogel. Furthermore, the porous 3D structure of the carbon aerogel remained unchanged after ten absorption/combustion cycles.

According to the results mentioned above, the absorption parameters were found to play an important role in determining the absorption capabilities of the CNF/PVA/GO carbon aerogels for removal of organic solvents and oils, including $\mathrm{N}, \mathrm{N}$ dimethylformamide (DMF), engine oil, gasoline, ethanol, corn germ oil, pump oil, soybean oil and used pump oil. As shown in Figure 11, the absorption capacity for all the organics and oils were above $57 \mathrm{~g} / \mathrm{g}$, especially for gasoline, which attained the highest value at $97 \mathrm{~g} / \mathrm{g}$. The absorption capacity and water contact angle of the CNF/PVA/GO carbon aerogel is higher than 


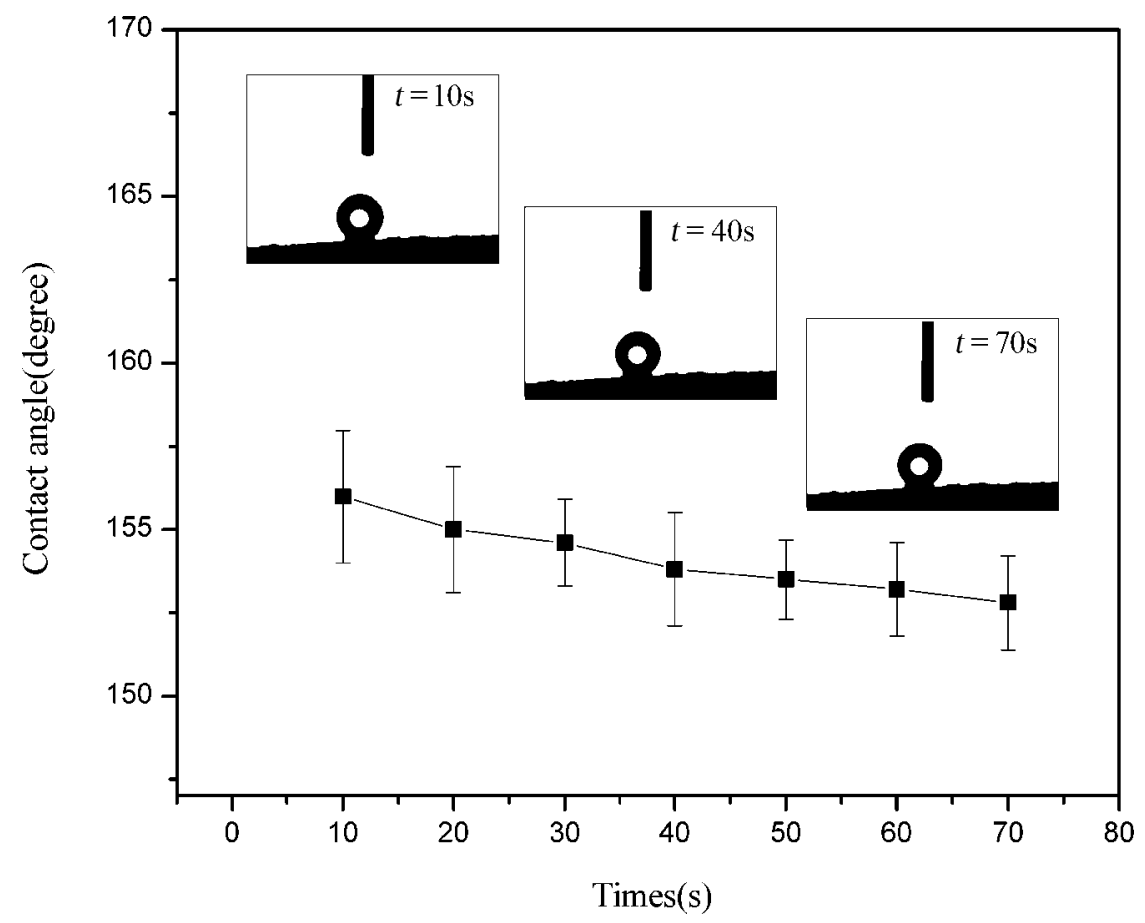

Figure 7: Contact angle of CNF/PVA/GO carbon aerogels.
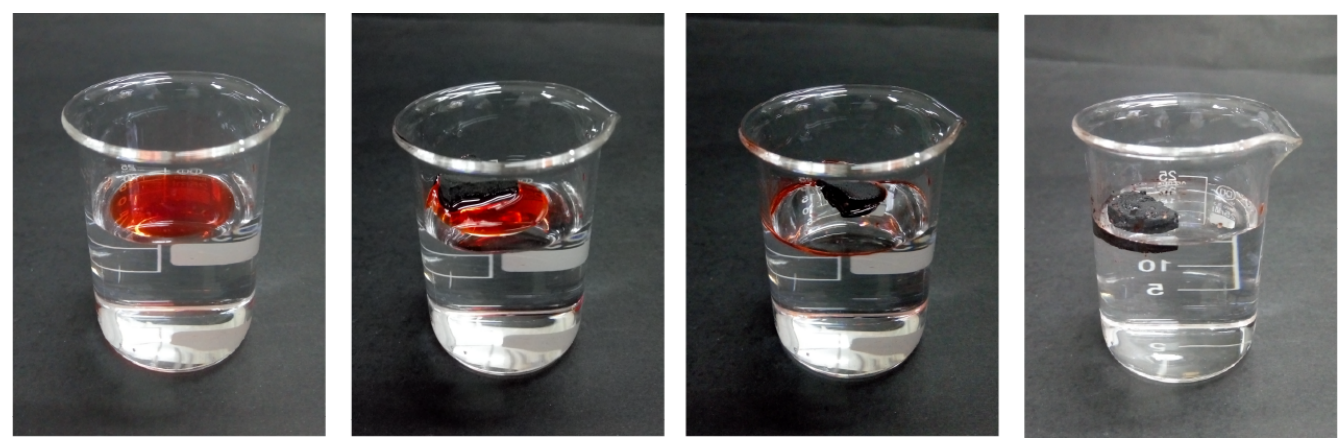

Figure 8: Removal of soybean oil (dyed with Sudan red) from the water surface using carbon aerogel.
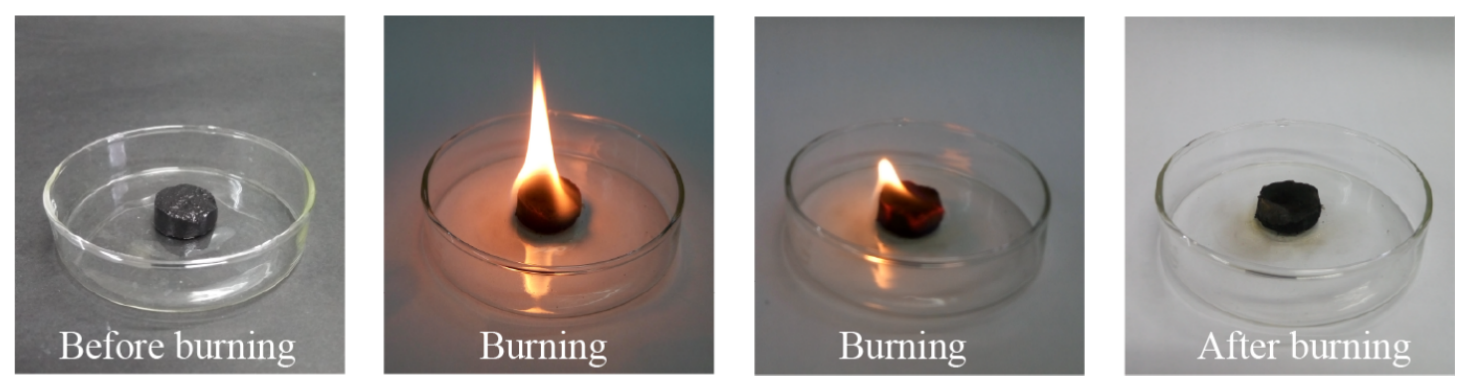

Figure 9: Photographs showing the recycling process of CNF/PVA/GO carbon aerogels via combustion. 


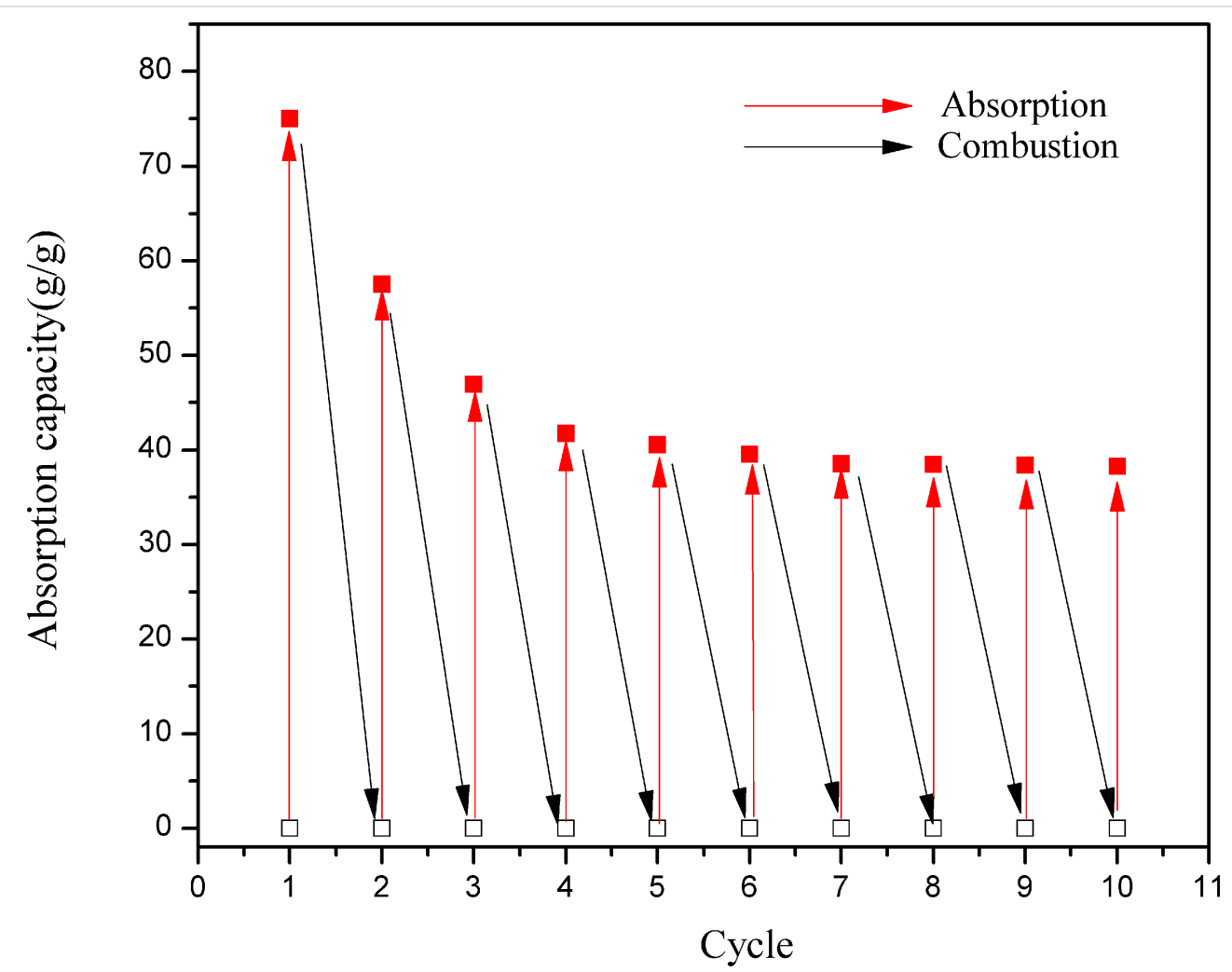

Figure 10: Recycling performance of CNF/PVA/GO carbon aerogels for absorption of soybean oil by absorption/combustion processes.

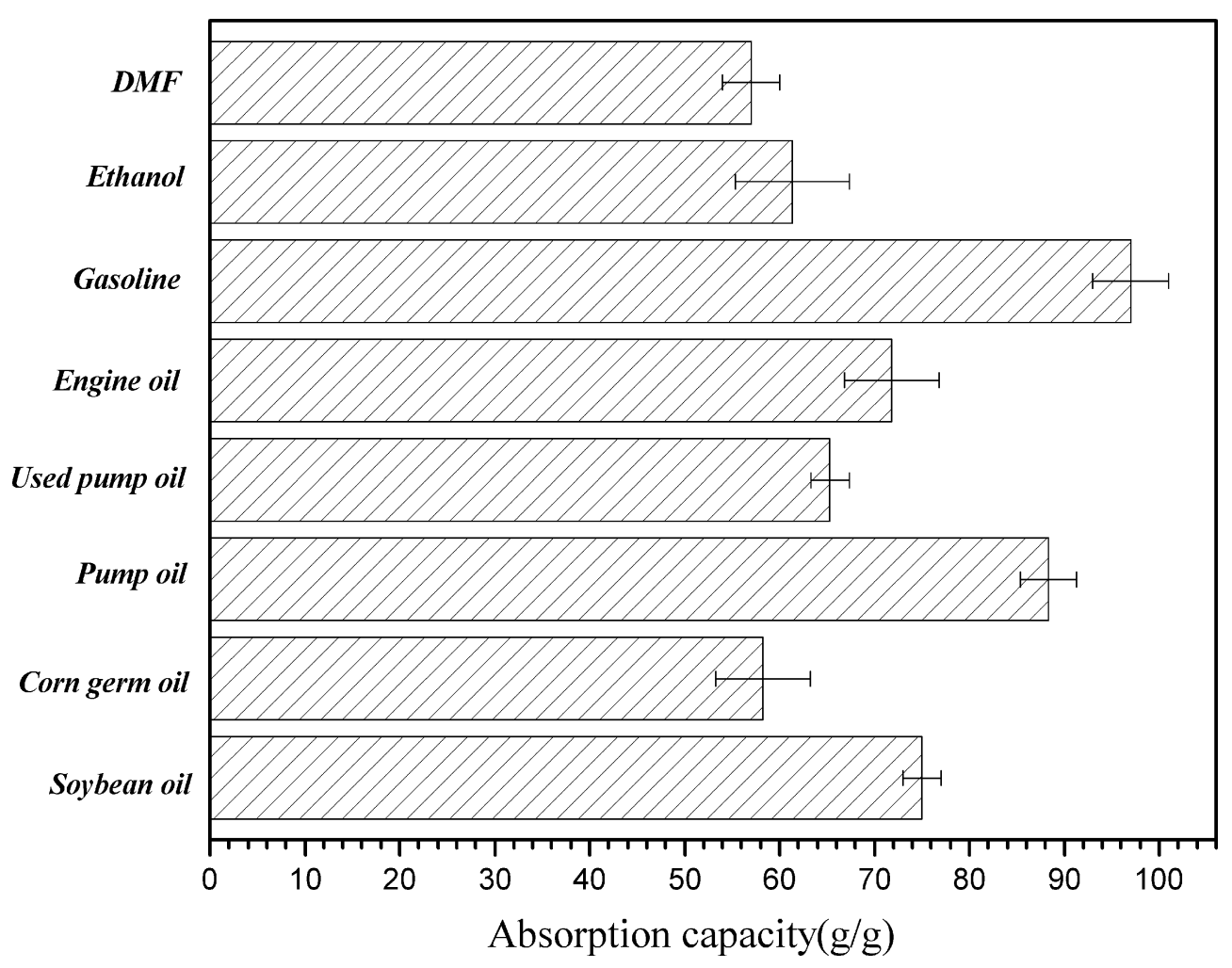

Figure 11: Absorption capacity of CNF/PVA/GO carbon aerogels. 
most existing oil-absorption materials (Table 1). This was attributed mainly to the strong capillary effect of the super-hydrophobic carbon aerogels with high porosity, small hole size and low surface energy. Obviously, the absorption capacity difference of the carbon aerogels was largely determined by the density of the carbon aerogel and surface tension of the solvents and oils. Additionally, the density, which demonstrates the quantity of the holes in the carbon aerogels, plays the more significant role in absorption capacity. The holes in the carbon aerogel provide sufficient space for the storage of absorbed organic solvents/oils, which increase carbon aerogel's absorption ability.

\section{Conclusion}

To summarize, a super-hydrophobic, lightweight and porous $\mathrm{CNF} / \mathrm{PVA} / \mathrm{GO}$ carbon aerogel has been prepared via a simple freeze-drying process and carbonization treatment. The obtained three-dimensional carbon aerogels exhibited a low density of $18.41 \mathrm{mg} \mathrm{cm}^{-3}$ and excellent super-hydrophobicity with a water contact angle of $156^{\circ}$. The super-hydrophobic CNF/PVA/ GO carbon aerogels exhibited a high absorption capacity of up to 97 times their own weight for oils and organic solvents. Furthermore, the carbon aerogel also exhibited high absorption selectivity, good recyclability and flame retardance. Our findings are a proof-of-concept for the use of carbonization treatment to provide hydrophobicity for the frame of three-dimension carbon aerogels and offers great potential for applications of these materials in treatment of oil pollution.

\section{Experimental}

\section{Materials}

Bamboo powder was used as the source for the preparation of cellulose and was purchased from Zhejiang Lishui, China. Graphite powder $(40 \mu \mathrm{m})$, used as the source for GO, was obtained from Qingdao Henglide Graphite Co., Ltd. Poly(vinyl alcohol) (PVA, $M_{\mathrm{w}} \approx 95,000 \mathrm{~g} / \mathrm{mol}$ ), glutaraldehyde (GA, crosslinker, $25 \mathrm{wt} \%$ in $\left.\mathrm{H}_{2} \mathrm{O}\right)$, potassium hydroxide $(\mathrm{KOH})$, Sudan III, acetic acid $\left(\mathrm{CH}_{3} \mathrm{COOH}\right)$, hydrogen peroxide $\left(\mathrm{H}_{2} \mathrm{O}_{2}\right.$,
$30 \%)$, potassium permanganate $\left(\mathrm{KMnO}_{4}\right)$, concentrated sulfuric acid $\left(\mathrm{H}_{2} \mathrm{SO}_{4}, 98 \%\right)$, hydrochloric acid $(\mathrm{HCl})$, sodium nitrate $\left(\mathrm{NaNO}_{3}\right)$, sodium chlorite $\left(\mathrm{NaClO}_{2}\right)$, phosphorus pentoxide $\left(\mathrm{P}_{2} \mathrm{O}_{5}\right)$, potassium persulfate $\left(\mathrm{K}_{2} \mathrm{~S}_{2} \mathrm{O}_{8}\right)$ and ammonium hydroxide $\left(\mathrm{NH}_{3} \cdot \mathrm{H}_{2} \mathrm{O}\right)$ were purchased from Nanjing Chemical Reagent Co., Ltd. These materials were all used without further purification.

\section{Preparation of carbon nanofibers}

The chemical treatment of bamboo powder was prepared by a modified method described in the literature [27]. The detailed experimental steps of preparing the cellulose suspension are documented in our previous paper [40]. Briefly, the suspensions were treated with $2 \mathrm{wt} \%$ aqueous $\mathrm{KOH}$ to remove pectin, hemicelluloses, and residual starch in the bamboo powder. Then, the samples were treated with a solution of $\mathrm{NaClO}_{2}$ and $\mathrm{CH}_{3} \mathrm{COOH}$ to remove lignin. Finally, the samples were treated with a $1 \mathrm{wt} \% \mathrm{HCl}$ solution to remove the excess metal ions. After the chemical treatment, the slurry of $1 \mathrm{wt} \%$ purified cellulose was ground by a grinder to obtain a CNF slurry [46].

\section{Preparation of graphene oxide}

GO was synthesized from graphite powder using a modified Hummers' method $[47,48]$. The fabrication process was recorded in detail in our previous research paper [40]. Then, we obtained exfoliated single-layered GO sheets in GO aqueous dispersions under ultrasonication at $80 \mathrm{kHz}$ for $2 \mathrm{~h}$.

\section{Preparation of poly(vinyl alcohol) solution}

PVA $(5.0 \mathrm{~g})$ was dissolved in deionized water $(100 \mathrm{~mL})$ with continuous stirring for $4 \mathrm{~h}$ at $85^{\circ} \mathrm{C}$ until the PVA was completely dissolved. Then, the PVA solution was sealed and stored at room temperature for further use.

\section{Fabrication of CNF/PVA/GO aerogel}

The CNF/PVA/GO aerogel preparation process is shown in Figure 12. The CNF solution (8.34 g, $1.2 \mathrm{wt} \%$ ), PVA solution ( $3 \mathrm{~mL}, 0.05 \mathrm{~g} \mathrm{~mL}^{-1}$ ), and GO solution (4.5 g, $3.34 \mathrm{wt} \%$ ) were

\begin{tabular}{|c|c|c|}
\hline Sorbant material & Absorption capacity (g/g) & Contact angle $\left({ }^{\circ}\right)$ \\
\hline CNF/PVA/GO carbon aerogels (this work) & $57-97$ & 156 \\
\hline silane-treated PVA/CNF aerogel [39] & 44-96 & 150 \\
\hline TMCS/rGO/CNF composite aerogel [40] & $33-39$ & 117 \\
\hline spongy graphene [41] & $20-86$ & 114 \\
\hline graphene-based aerogel [42] & $28-40$ & - \\
\hline MTMS-coated cellulose aerogels [43] & $18-20$ & 135 \\
\hline super-hydrophobic kapok fiber [44] & $54.2-59.8$ & 151 \\
\hline polyurethane/polysiloxane sponge [45] & $15-25$ & 157 \\
\hline
\end{tabular}




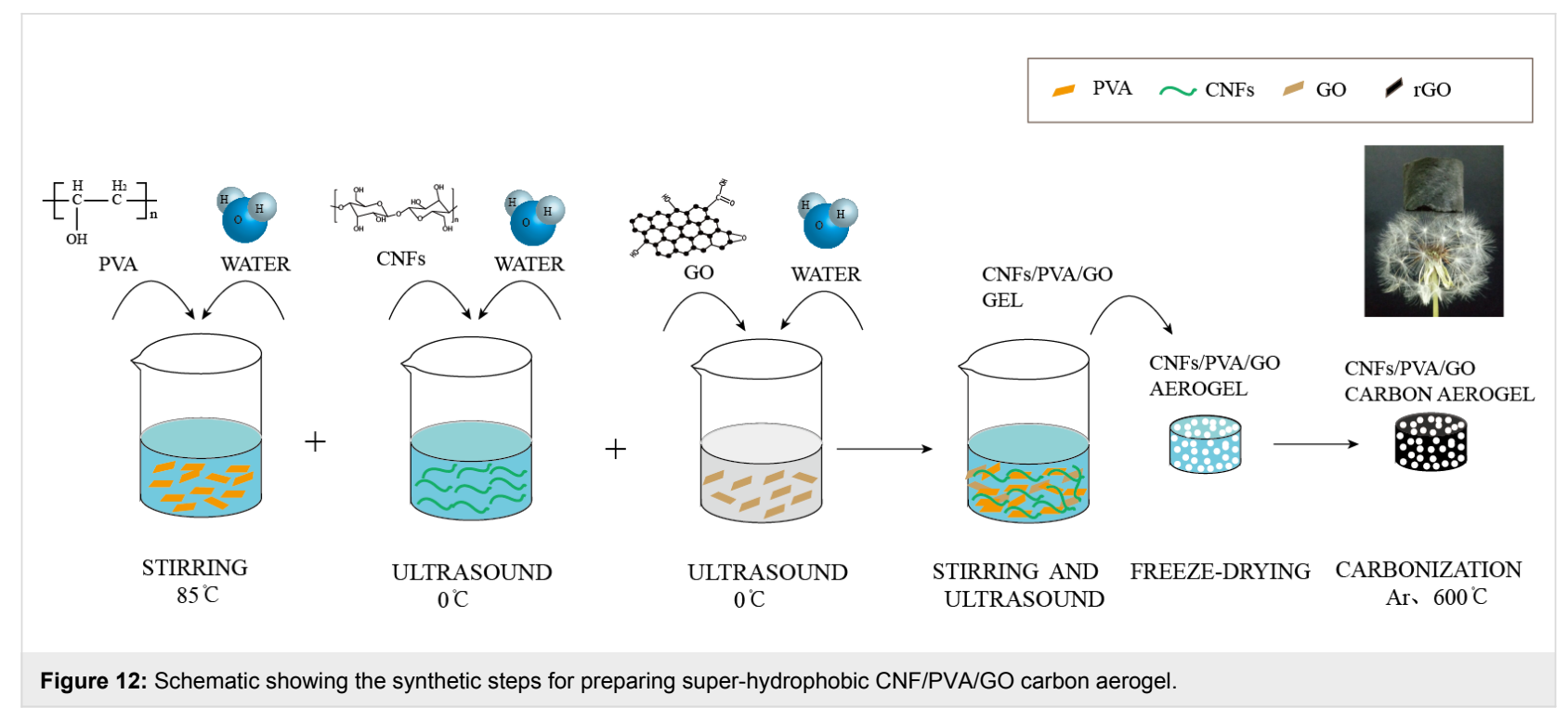

mixed together by vigorous stirring for $1 \mathrm{~h}$. Sulfuric acid $(0.8 \mathrm{~mL}, 1.0 \mathrm{vol} \%)$ was then added to the CNF/PVA/GO solution with $\mathrm{pH}$ of approximately 5 . Then, a glutaraldehyde solution $(0.8 \mathrm{~mL}, 25 \mathrm{wt} \%)$ was added to the resulting $\mathrm{CNF} / \mathrm{PVA} /$ GO solution with mechanical stirring for $1 \mathrm{~h}$, and the mixture was then sonicated in an ultrasonic bath for another hour. Then, the solution was placed in a water bath at $35^{\circ} \mathrm{C}$ for $30 \mathrm{~min}$ to remove any residual air bubbles. The obtained aqueous gel was subsequently cross-linked/cured in an oven at $75^{\circ} \mathrm{C}$ for $3 \mathrm{~h}$. The cross-linked/cured aqueous gel was stored in a refrigerator overnight until completely frozen. Finally, the CNF/PVA/GO aerogels were prepared by a freeze drier.

\section{Fabrication of super-hydrophobic CNF/PVA/ GO carbon aerogel}

The carbonization treatment was performed by transferring the obtained CNF/PVA/GO aerogel into a tubular furnace. Firstly, argon gas was introduced for 10 min to remove the air in the $\mathrm{CNF} / \mathrm{PVA} / \mathrm{GO}$ aerogel and tubular furnace. Next, the furnace was heated to $200{ }^{\circ} \mathrm{C}$ at a heating rate of $4{ }^{\circ} \mathrm{C} \mathrm{min}{ }^{-1}$ and sustained for $30 \mathrm{~min}$. Then, it was heated to $600^{\circ} \mathrm{C}$ at a rate of $3{ }^{\circ} \mathrm{C} \mathrm{min}{ }^{-1}$ and held at this temperature for $2 \mathrm{~h}$ to assure complete pyrolysis. The following step was to cool it to $200{ }^{\circ} \mathrm{C}$ at a rate of $4{ }^{\circ} \mathrm{C} \mathrm{min}-1$, and finally, natural cooling to room temperature $\left(25^{\circ} \mathrm{C}\right)$ to yield a black and super-hydrophobic $\mathrm{CNF} /$ PVA/GO carbon aerogel.

\section{Sample characterization}

A scanning electron microscope (SEM, Quanta 200, FEI, USA) equipped with an energy disperse spectroscopy (EDS) device was used to investigate the surface morphology of the carbon aerogels. The FTIR profiles were obtained using a Fourier transform infrared spectroscopy spectrometer (Nicolet iS10,
Thermo Electron Corp., USA). The water contact angle (CA) of the carbon aerogels was obtained using an optical contact angle meter (OCA, Data Physics) with $4 \mu \mathrm{L}$ water droplets. Raman spectra results were measured using a DXR Raman spectrophotometer (Thermo Scientific, USA). Thermal stability measurements were measured using a thermogravimetric analyzer (TGA, Q 50 TA Instruments, USA). The X-ray photoelectron spectrometry spectra results were carried out using X-ray photoelectron spectroscopy (XPS, AXIS UltraDLD, USA).

The absorption capacity of the CNF/PVA/GO carbon aerogels was measured by immersing the carbon aerogels into various organic reagents and oils for $10 \mathrm{~min}$ until saturation. The wet carbon aerogels used for oil absorption were removed from the solution, lifted up for $30 \mathrm{~s}$ to allow the surface residual liquid to drip away, and then weighed. The absorption capacity of the carbon aerogels is calculated according to:

$$
C=\frac{W_{2}-W_{1}}{W_{1}}
$$

where $C$ is the absorption capacity of the carbon aerogels and $W_{1}$ and $W_{2}$ are the weights of the carbon aerogel before and after absorption. The porosity of aerogel samples was calculated using the following formula:

$$
\varphi=\left(1-\frac{\rho_{\mathrm{a}}}{\rho_{\mathrm{c}}}\right) \times 100
$$

where $\varphi$ is the porosity of the carbon aerogels, $\rho_{\mathrm{a}}$ is the density of the carbon aerogels and $\rho_{c}$ is the density of cellulose $\left(1.5 \mathrm{~g} \mathrm{~cm}^{-3}\right)$ [9]. 


\section{Acknowledgements}

This work was supported by the National Natural Science Foundation of China (grant no. 31770607), the National Key Research and Development Program of China (2017YFD0600204), the Natural Science Foundation of Jiangsu Province of China (grant no. BK20171450) and a project funded by the Priority Academic Program Development of Jiangsu Higher Education Institutions (PAPD). The authors acknowledge the Advanced Analysis \& Testing Center of Nanjing Forestry University.

\section{ORCID ${ }^{\circledR}$ iDs}

Jiangtao Shi - https://orcid.org/0000-0002-5469-4488

\section{References}

1. Hubbe, M. A.; Rojas, O. J.; Fingas, M.; Gupta, B. S. BioResources 2013, 8, 3038-3097. doi:10.15376/biores.8.2.3038-3097

2. Zhu, L.; Wang, Y.; Wang, Y.; You, L.; Shen, X.; Li, S. Microporous Mesoporous Mater. 2017, 241, 285-292. doi:10.1016/j.micromeso.2016.12.033

3. Bayat, A.; Aghamiri, S. F.; Moheb, A.; Vakili-Nezhaad, G. R. Chem. Eng. Technol. 2005, 28, 1525-1528. doi:10.1002/ceat.200407083

4. Wu, Z.-Y.; Li, C.; Liang, H.-W.; Zhang, Y.-N.; Wang, X.; Chen, J.-F.; Yu, S.-H. Sci. Rep. 2014, 4, 4079. doi:10.1038/srep04079

5. Klemm, D.; Heublein, B.; Fink, H.-P.; Bohn, A. Angew. Chem., Int. Ed. 2005, 44, 3358-3393. doi:10.1002/anie.200460587

6. Klemm, D.; Kramer, F.; Moritz, S.; Lindström, T.; Ankerfors, M.; Gray, D.; Dorris, A. Angew. Chem., Int. Ed. 2011, 50, 5438-5466. doi:10.1002/anie.201001273

7. Moon, R. J.; Martini, A.; Nairn, J.; Simonsen, J.; Youngblood, J. Chem. Soc. Rev. 2011, 40, 3941-3994. doi:10.1039/c0cs00108b

8. Iwamoto, S.; Kai, W.; Isogai, A.; Iwata, T. Biomacromolecules 2009, 10, 2571-2576. doi:10.1021/bm900520n

9. Cervin, N. T.; Aulin, C.; Larsson, P. T.; Wågberg, L. Cellulose 2012, 19, 401-410. doi:10.1007/s10570-011-9629-5

10. Li, S.-M.; Jia, N.; Ma, M.-G.; Zhang, Z.; Liu, Q.-H.; Sun, R.-C. Carbohydr. Polym. 2011, 86, 441-447. doi:10.1016/j.carbpol.2011.04.060

11. Eichhorn, S. J.; Dufresne, A.; Aranguren, M.; Marcovich, N. E.; Capadona, J. R.; Rowan, S. J.; Weder, C.; Thielemans, W.; Roman, M.; Renneckar, S.; Gindl, W.; Veigel, S.; Keckes, J.; Yano, H.; Abe, K.; Nogi, M.; Nakagaito, A. N.; Mangalam, A.; Simonsen, J.; Benight, A. S.; Bismarck, A.; Berglund, L. A.; Peijs, T. J. Mater. Sci. 2010, 45, 1. doi:10.1007/s10853-009-3874-0

12. Miao, C.; Hamad, W. Y. Cellulose 2013, 20, 2221-2262. doi:10.1007/s10570-013-0007-3

13. Javadi, A.; Srithep, Y.; Pilla, S.; Lee, J.; Gong, S.; Turng, L.-S. Mat. Sci. Eng. C 2010, 30, 749-757. doi:10.1016/j.msec.2010.03.008

14. Voisin, H.; Bergström, L.; Liu, P.; Mathew, A. P. Nanomaterials 2017, 7, 57. doi:10.3390/nano7030057

15. Abdul Khalil, H. P. S.; Bhat, A. H.; Ireana Yusra, A. F. Carbohydr. Polym. 2012, 87, 963-979. doi:10.1016/j.carbpol.2011.08.078

16. Siró, I.; Plackett, D. Cellulose 2010, 17, 459-494. doi:10.1007/s10570-010-9405-y
17. Nakagaito, A. N.; Yano, H. Cellulose 2008, 15, 555-559. doi:10.1007/s10570-008-9212-x

18. Henriksson, M.; Berglund, L. A. J. Appl. Polym. Sci. 2007, 106, 2817-2824. doi:10.1002/app.26946

19. Han, S.; Sun, Q.; Zheng, H.; Li, J.; Jin, C. Carbohydr. Polym. 2016, 136, 95-100. doi:10.1016/j.carbpol.2015.09.024

20. Wu, Q.; Henriksson, M.; Liu, X.; Berglund, L. A. Biomacromolecules 2007, 8, 3687-3692. doi:10.1021/bm701061t

21. Malainine, M. E.; Mahrouz, M.; Dufresne, A. Compos. Sci. Technol. 2005, 65, 1520-1526. doi:10.1016/j.compscitech.2005.01.003

22. Wang, B.; Sain, M. Polym. Int. 2007, 56, 538-546. doi:10.1002/pi.2167

23. Samir, M. A. S. A.; Alloin, F.; Sanchez, J. Y.; Dufresne, A. Polymer 2004, 45, 4149-4157. doi:10.1016/j.polymer.2004.03.094

24. Kowalczyk, M.; Piorkowska, E.; Kulpinski, P.; Pracella, M. Composites, Part A 2011, 42, 1509-1514. doi:10.1016/j.compositesa.2011.07.003

25. Matsumura, S.; Kurita, H.; Shimokobe, H. Biotechnol. Lett. 1993, 15 , 749-754. doi:10.1007/BF01080150

26. Yao, X.; Yu, W.; Xu, X.; Chen, F.; Fu, Q. Nanoscale 2015, 7, 3959-3964. doi:10.1039/C4NR07402E

27. Chen, W.; Yu, H.; Liu, Y. Carbohydr. Polym. 2011, 86, 453-461. doi:10.1016/j.carbpol.2011.04.061

28. Wang, S.; Ren, J.; Li, W.; Sun, R.; Liu, S. Carbohydr. Polym. 2014, 103, 94-99. doi:10.1016/j.carbpol.2013.12.030

29. Meng, Y.; Young, T. M.; Liu, P.; Contescu, C. I.; Huang, B.; Wang, S. Cellulose 2015, 22, 435-447. doi:10.1007/s10570-014-0519-5

30. Adebajo, M. O.; Frost, R. L.; Kloprogge, J. T.; Kokot, S. Spectrochim. Acta, Part A: Mol. Biomol. Spectrosc. 2006, 64, 448-453. doi:10.1016/j.saa.2005.07.045

31. Ferrari, A. C.; Robertson, J. Phys. Rev. B 2000, 61, 14095. doi:10.1103/PhysRevB.61.14095

32. Ren, P.-G.; Yan, D.-X.; Chen, T.; Zeng, B.-Q.; Li, Z.-M. J. Appl. Polym. Sci. 2011, 121, 3167-3174. doi:10.1002/app.33856

33. Paredes, J. I.; Villar-Rodil, S.; Solís-Fernández, P.; Martínez-Alonso, A.; Tascón, J. M. D. Langmuir 2009, 25, 5957-5968. doi:10.1021/la804216z

34. Zhang, H.-B.; Wang, J.-W.; Yan, Q.; Zheng, W.-G.; Chen, C.; Yu, Z.-Z. J. Mater. Chem. 2011, 21, 5392-5397. doi:10.1039/c1jm10099h

35. Yang, S. J.; Kim, T.; Jung, H.; Park, C. R. Carbon 2013, 53, 73-80. doi:10.1016/j.carbon.2012.10.032

36. Jeong, H.-K.; Lee, Y. P.; Jin, M. H.; Kim, E. S.; Bae, J. J.; Lee, Y. H. Chem. Phys. Lett. 2009, 470, 255-258. doi:10.1016/j.cplett.2009.01.050

37. Jeong, H.-K.; Lee, Y. P.; Lahaye, R. J. W. E.; Park, M.-H.; An, K. H.; Kim, I. J.; Yang, C.-W.; Park, C. Y.; Ruoff, R. S.; Lee, Y. H. J. Am. Chem. Soc. 2008, 130, 1362-1366. doi:10.1021/ja076473o

38. Pham, C. V.; Eck, M.; Krueger, M. Chem. Eng. J. 2013, 231, 146-154. doi:10.1016/j.cej.2013.07.007

39. Zheng, Q.; Cai, Z.; Gong, S. J. Mater. Chem. A 2014, 2, 3110-3118. doi:10.1039/C3TA14642A

40. Xu, Z.; Zhou, H.; Jiang, X.; Li, J.; Huang, F. IET Nanobiotechnol. 2017, 11, 929-934. doi:10.1049/iet-nbt.2017.0063

41. Bi, H.; Xie, X.; Yin, K.; Zhou, Y.; Wan, S.; He, L.; Xu, F.; Banhart, F.; Sun, L.; Ruoff, R. S. Adv. Funct. Mater. 2012, 22, 4421-4425. doi:10.1002/adfm.201200888

42. Wu, T.; Chen, M.; Zhang, L.; Xu, X.; Liu, Y.; Yan, J.; Wang, W.; Gao, J. J. Mater. Chem. A 2013, 1, 7612-7621. doi:10.1039/c3ta10989e

43. Nguyen, S. T.; Feng, J.; Ng, S. K.; Wong, J. P.; Tan, V. B. C.; Duong, H. M. Colloids Surf., A 2014, 445, 128-134. doi:10.1016/j.colsurfa.2014.01.015 
44. Wang, J.; Zheng, Y.; Wang, A. Chem. Eng. J. 2012, 213, 1-7. doi:10.1016/j.cej.2012.09.116

45. Zhu, Q.; Chu, Y.; Wang, Z.; Chen, N.; Lin, L.; Liu, F.; Pan, Q. J. Mater. Chem. A 2013, 1, 5386-5393. doi:10.1039/c3ta00125c

46. Abe, K.; Yano, H. Cellulose 2010, 17, 271-277. doi:10.1007/s10570-009-9382-1

47. Hoai, N. T.; Sang, N. N.; Hoang, T. D. Mater. Sci. Eng., B 2017, 216 , 10-15. doi:10.1016/j.mseb.2016.06.007

48. Hummers, W. S., Jr.; Offeman, R. E. J. Am. Chem. Soc. 1958, 80, 1339. doi:10.1021/ja01539a017

\section{License and Terms}

This is an Open Access article under the terms of the Creative Commons Attribution License

(http://creativecommons.org/licenses/by/4.0), which permits unrestricted use, distribution, and reproduction in any medium, provided the original work is properly cited.

The license is subject to the Beilstein Journal of

Nanotechnology terms and conditions:

(https://www.beilstein-journals.org/bjnano)

The definitive version of this article is the electronic one which can be found at: doi:10.3762/bjnano.9.49 